\title{
Decompression alone versus decompression with limited fusion for treatment of degenerative lumbar scoliosis in the elderly patient
}

Authors Michael D Daubs ${ }^{1}$, Lawrence G Lenke², Keith H Bridwell ${ }^{2}$, Gene Cheh ${ }^{3}$, Yongjung J Kim ${ }^{4}$, Georgia Stobbs ${ }^{2}$

Institutions $\quad{ }^{1}$ Department of Orthopaedic and Neurosurgery, University of California, Los Angeles, USA

${ }^{2}$ Department of Orthopaedic Surgery, Washington University School of Medicine,

St Louis, MO, USA

${ }^{3}$ Department of Orthopaedic Surgery, Wooridul Spine Hospital, Seoul, South Korea

${ }^{4}$ Department of Orthopaedic Surgery, Columbia University, New York, USA

\begin{tabular}{l|l|}
\hline Final class of \\
evidence-prognosis \\
Study design \\
\hline RCT \\
Cohort \\
Case control \\
Case series \\
\hline Methods \\
\hline Concealed allocation (RCT) \\
\hline Intention to treat (RCT) \\
\hline Blinded/independent \\
evaluation of primary outcome
\end{tabular}

\begin{abstract}
Study design: Retrospective cohort study.

Objective: To analyze the surgical results of a group of patients older than 65 years treated for mild degenerative lumbar scoliosis $\left(<30^{\circ}\right)$ with stenosis, treated with decompression alone or decompression and limited fusion.

Methods: We evaluated 55 patients, all older than 65 years from our prospectively collected database with mild degenerative scoliosis $\left(<30^{\circ}\right)$ and stenosis who underwent surgery. Laminectomy alone was performed in 16 patients, and laminectomy and limited fusion in 39 patients. Mean followup was 4.6 years in the decompression group and 5.0 years in the fusion group. Clinical results were graded by patients' self-reported satisfaction and length of symptom-free period to recurrence.
\end{abstract}

Results: In the decompression alone group, 6 (37\%) of 16 patients developed recurrent stenosis at the previously decompressed level and five developed recurrence within 6 months postoperatively versus the decompression and fusion group where $3(8 \%)$ of $39(P=.0476)$ developed symptomatic stenosis supra adjacent to the fusion. Of 16 patients in the decompression alone group, $12(75 \%)$ had recurrence of symptoms by the 5 -year follow-up period versus only $14(36 \%)$ patients in the decompression and fusion group $(P=.016)$. Adjacent segment degenerative changes were common in the fusion group, but only $7 \%$ developed symptomatic stenosis.

Conclusions: Decompression with limited fusion prevents early return of stenotic symptoms compared with decompression alone in the setting of mild degenerative scoliosis $\left(<30^{\circ}\right)$ and symptomatic stenosis in patients 65 years and older. 


\section{STUDY RATIONALE AND CONTEXT}

The surgical treatment of degenerative lumbar scoliosis with stenosis (DLS) in the elderly is challenging [1-5]. The goal is to reduce surgical morbidity and achieve good, longstanding results [6]. In patients who are not considered candidates for a long thoracic and lumbar fusion, decompression alone and decompression with limited fusion are the main treatment options. There are few reports evaluating older patients with DLS.

\section{OBJECTIVE}

To compare the surgical treatment results of decompression only to decompression and fusion in patients older than 65 years with mild degenerative scoliosis $\left(<30^{\circ}\right)$ and symptomatic stenosis consisting of radiculopathy or neurogenic claudication.

Fig 1 Patient sampling and selection.

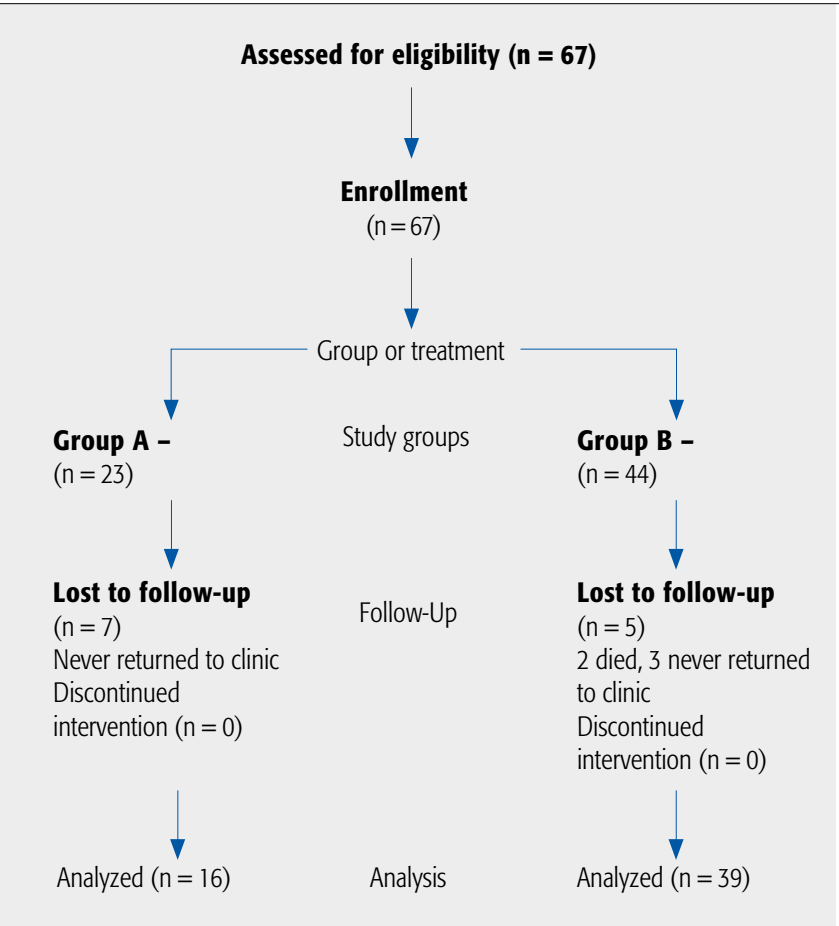

\section{METHODS}

Study design: Retrospective cohort study.

\section{Inclusion criteria:}

- Patients older than 65 years with degenerative scoliosis

- Degenerative scoliosis less than $30^{\circ}$

- Lumbar stenosis with radiculopathy or symptoms of neurogenic claudication refractory to non-surgical treatment

- Patients with a minimum 24-month follow-up

\section{Exclusion criteria:}

- Patients younger than 65 years

- Degenerative scoliosis greater than $30^{\circ}$

- Patients without radiculopathy or neurogenic claudication symptoms

Patient population and selection: Fifty-five consecutive patients with DLS and stenosis who underwent surgery by two spine surgeons at one institution from 1996-2003 were retrospectively reviewed radiographically and clinically. All patients were treated during the same period. The standard of care at our institution for the treatment of degenerative scoliosis of less than $30^{\circ}$ with stenosis is laminotomy/ laminectomy without fusion, or laminotomy/laminectomy with limited fusion at the levels of decompression only.

\section{Surgical technique:}

- The technique for the decompression only (group D) consisted of a standard bilateral laminotomy and foraminotomy or hemilaminectomy with an effort to preserve at least $50 \%$ of the facet bilaterally.

- The technique for decompression and fusion (group DF) included a similar technique for decompression as previously described, as well as the addition of an instrumented fusion. The instrumented fusion consisted of the placement of pedicle screw instrumentation at the level or levels decompressed, followed by the placement of a connecting rod. The rod was bent to match the curve on the table and no active corrective maneuvers were used. Iliac crest and local autograft was used for the posterior intertransverse arthrodesis. 


\section{Outcomes:}

The following outcomes were measured:

- Length of symptom-free time: This data was gathered from the medical record at follow-up intervals ( 6 weeks, 3 months, 6 months, 12 months, and 24 months). If the patients began to have recurrent symptoms before the routine follow-up period, and the specific time was noted, this was recorded.

- Global clinical assessment (Table1): This non-validated clinical assessment was developed by the authors to evaluate the outcomes. The clinical assessment category was determined from written information contained in the medical record. The clinical assessment category was determined by an independent surgeon who was not one of the two senior treating surgeons. The reviewer was not blinded to the treatment. An 'excellent' outcome was identified as a patient with complete relief of his/her radicular or neurogenic symptoms. A good result was defined when symptoms were improved without recurrent radicular symptoms, but not overall completely symptom free. A fair result was defined as one with mild symptoms of recurrence similar to preoperative symptoms, but improved compared with preoperative condition. A poor result was recurrence of preoperative symptoms, little improvement, or symptoms that were unchanged.

- Radiographic Cobb angle change: The global clinical assessment and radiographic Cobb angle measurements were recorded at the 2-year period for all patients and at the last follow-up for patients with 5-year follow-up.

- Radiographic adjacent segment degeneration: A diagnosis of radiographic adjacent segment degeneration (ASD) was assigned when there was $>75 \%$ disc space narrowing, large spur formation, olisthesis or translation $\geq 5 \mathrm{~mm}$ at the disc level adjacent to the fused segments [8] or when there were symptoms of spinal stenosis (neurogenic claudication or radiculopathy) referable to the adjacent segment and confirmed by selective nerve root injections.

\section{ANALYSIS}

- Unpaired $t$ tests were performed to analyze the demographic differences between patients who underwent a decompression alone and those who underwent a decompression and limited fusion.

- Unpaired $t$ tests were also used to evaluate the differences in length of symptom-free time.

- Fisher exact test was used to determine the differences in the global assessment and the presence of ASD.

- A paired $t$ test was used to evaluate the differences in radiographic Cobb angles within each subject.

Table 1 Clinical results at 2-year follow-up.

\begin{tabular}{lllll}
\hline & & $\begin{array}{l}\text { Decompression } \\
\text { alone } \mathbf{( n = 1 6 )} \\
\text { No. (\%) }\end{array}$ & $\begin{array}{l}\text { Decompression/ } \\
\text { limited fusion } \\
\mathbf{( n = 3 9 )} \\
\text { No. (\%) }\end{array}$ & P* \\
\hline Excellent & Symptom relief & $9(56)$ & $28(72)$ & .16 \\
\hline Good & $\begin{array}{l}\text { Minor residual } \\
\text { symptoms } \\
\text { No recurrence, } \\
\text { better than } \\
\text { preoperative }\end{array}$ & $4(10)$ & \\
\hline Fair & $\begin{array}{l}\text { Recurrence, } \\
\text { better than } \\
\text { preoperative }\end{array}$ & & \\
\hline Poor & $\begin{array}{l}\text { Recurrence, } \\
\text { unchanged or } \\
\text { worse than } \\
\text { preoperative }\end{array}$ & & \\
\hline
\end{tabular}

* Comparisons were made using a Fisher exact test. Two groups were compared: excellent/good; fair/poor. 


\section{RESULTS}

- Mean age was 75 years in the decompression group (D) and 66 years in the limited fusion group (DF) $(P=.01)$. Mean follow-up was 4.6 years in the decompression group and 4.6 years in the fusion group (Table 2).

- Hundred percent of patients had follow-up at the 2-year follow-up.

- Twenty-five (45\%) of 55 patients had 5-year follow-up.

- Overall, satisfactory (good-to-excellent) results were found in $10(63 \%)$ of 16 in Group D and $32(82 \%)$ of 39 in Group DF at 2-year follow-up $(P=.16)$.

- In Group D, 10 (63\%) of 16 patients remained symptom free at 2-year follow-up, and in Group DF, 36 $(92 \%)$ of 39 patients remained asymptomatic at 2 years $(P=.018)$ (Table 3).

- In Group D, 4 (25\%) of 16 had evidence of ASD while in Group DF 20 (51\%) of 39 patients had evidence of $\operatorname{ASD}(P=.13)$.

- In Group D, the mean preoperative Cobb angle was $16^{\circ}$, and $18^{\circ}$ at last follow-up for a mean change of $2^{\circ}$ (range, $0-8^{\circ}$ ), and in Group DF, the mean Cobb angle did not change from preoperative to last follow-up at a value of $22^{\circ}$ for a mean change of $0^{\circ}$ (range, $0-17^{\circ}$ ) $(P=.17)$.

- There were 25 patients overall $(47 \%)$ with at least 5-year follow-up, 8 (50\%) in Group D and 17 (43\%) in Group DF. At 5-year follow-up, 4 (50\%) of 8 patients in Group D had satisfactory (good) results and $3(37 \%)$ of 8 had evidence of ASD. In Group DF, 10 (59\%) of 17 had satisfactory (good) results $(P=1.0)$, and $14(82 \%)$ of 17 showed disc space collapse at the supra-adjacent segment.

- Of 16 patients in the decompression alone group, 12 (75\%) had recurrence of symptoms by the 5 -year follow-up versus only $14(36 \%)$ in the decompression and fusion group $(P=.016)$.

- Complications are listed in Table 4.
Table 2 Demographics and radiographic data.

\begin{tabular}{|c|c|c|c|}
\hline & $\begin{array}{l}\text { Decompression } \\
\text { alone }(n=16)\end{array}$ & $\begin{array}{l}\text { Decompression/ } \\
\text { limited fusion } \\
(n=39)\end{array}$ & $\boldsymbol{P}$ \\
\hline Average age, y & $75 \pm 6$ & $66 \pm 7$ & $.01^{*}$ \\
\hline Average follow-up, y & $4.6( \pm 2.7)$ & $4.6( \pm 2.4)$ & $.39 *$ \\
\hline $\begin{array}{l}\text { Average preoperative } \\
\text { lumbar scoliosis, }\end{array}$ & $16 \pm 6$ & $22 \pm 8$ & $.23^{*}$ \\
\hline $\begin{array}{l}\text { Average postoperative } \\
\text { correction, }^{\circ}\end{array}$ & $3 \pm 4$ & $1 \pm 8$ & $.5 *$ \\
\hline Levels treatedt & & & $.74 \dagger$ \\
\hline 1 & 5 & 12 & \\
\hline 2 & 9 & 27 & \\
\hline 3 & 1 & & \\
\hline 4 & 1 & & \\
\hline
\end{tabular}

* Unpaired $t$ test.

† Fisher exact test.

Table 3 Time interval of recurrent leg pain.

\begin{tabular}{llll}
\hline $\begin{array}{l}\text { Recurrent spinal } \\
\text { stenosis }\end{array}$ & $\begin{array}{l}\text { Decompression } \\
\text { alone } \mathbf{n = 1 6}(\mathbf{1 \% )}\end{array}$ & $\begin{array}{l}\text { Decompression/ } \\
\text { limited fusion } \\
\mathbf{n = 3 9}(\%)\end{array}$ & $\boldsymbol{P}^{*}$ \\
\hline Within first 6 mo & $5(31)$ & $0(0)$ & .001 \\
\hline Between 6 mo and 2y & $1(9)$ & $3(7)$ & 1.00 \\
\hline Within 2-y follow-up & $6(37)$ & $3(8)$ & .018 \\
\hline
\end{tabular}

* Comparisons were made using Fisher exact test.

Table 4 Complications.

\begin{tabular}{llll}
\hline & $\begin{array}{l}\text { Decompression } \\
\text { alone } \mathbf{n = 1 6}(\mathbf{1 \% )}\end{array}$ & $\begin{array}{l}\text { Decompression/ } \\
\text { limited fusion } \\
\mathbf{n = 3 9}(\%)\end{array}$ & $\boldsymbol{P}^{\boldsymbol{\dagger}}$ \\
\hline Deep vein thrombosis & $0(0)$ & $1(2)$ & 1.00 \\
\hline Dural tear & $1(6)$ & $1(2)$ & .50 \\
\hline Deep wound infection & $0(0)$ & $2(5)$ & 1.00 \\
\hline $\begin{array}{l}\text { Reoperation } \\
\text { - Decompression } \\
\text { - Fusion }\end{array}$ & $1(6)$ & $2 *(5)$ & .053 \\
\hline
\end{tabular}

* Two additional patients are candidates for revision surgery.

† Comparisons were made using Fisher exact test. 


\section{DISCUSSION}

- A significant number of patients who had decompression without fusion had recurrent symptoms $(37 \%)$ at the same level within the 2-year follow-up versus only $8 \%$ in the decompression and fusion group $(P=.018)$ (Table 3).

- Decompression and fusion had more sustainable results in regard to recurrence of symptoms at 2 years and at final follow-up compared with decompression alone.

- In this consecutive series of patients, there was selection bias in determining the type of surgical treatment offered to patients with DLS and stenosis. Patients who underwent a decompression alone were significantly older in age compared with those with decompression and limited fusion.

- Progression of scoliosis was not seen in our patient population regardless of the type of treatment. The treatment of symptomatic stenosis in well-selected patients with mild scoliosis $<30^{\circ}$ with decompression or decompression and limited fusion does not appear to destabilize the spine enough to cause curve progression.

- With further follow-up (5 years), there was still no evidence of curve progression in either group; however, the fusion group had a higher incidence of ASD at the supra-adjacent level $[7,8]$, and in general, the results of both treatment methods deteriorated with time.

- This is a difficult patient population to treat and there are no clear standards [1-5]. As this series shows, we tend to treat older patients with the less invasive procedure but the symptoms often quickly recur leaving doubt to its ultimate effectiveness. If we add fusion, we seem to lengthen the symptom-free period.

- The surgeons in this series did not have a formal algorithm that guided their treatment. However, they are both experienced spinal deformity surgeons with an awareness of the potential for curve progression with decompression alone. While age was significantly different between the treatment groups, this was only recognized after the statistical analysis. In general, patients at our institution who undergo a decompression alone will have large stabilizing osteophytes, severe disc space collapse, and mild scoliotic curves $<30^{\circ}$.

- There are many confounding factors in this study that are inherent to its retrospective nature and may have impacted the results. The fact that both senior surgeons are experienced in treating deformity and may be better at selecting patients who are more appropriate for decompression alone may have had an impact on the results.

- While there was no significant curve progression seen in this study, there was significant recurrence of ste- notic symptoms in the decompression alone group. So, it would appear that the surgeons were able to accurately assess patients whose curves were stable enough for decompression alone, but were not able to predict in whom symptoms might recur. It appears that symptoms can recur without noticeable curve progression. One can speculate that more subtle changes in rotation and translation may be occurring that are not detected in standard Cobb measurements.

- As this study shows, there is inherent bias toward treating older patients with the least invasive procedure (decompression alone) but if the goal is to provide the treatment that gives the longest and most sustainable results, less in these cases may be more. Adding a limited fusion to the decompressed segments seems to improve the length of symptom-free time.

- There are many limitations to this study. It is a retrospective case series with many inherent treatment biases and confounding factors that may have affected the results. The smaller number of patients included for comparison that underwent a decompression alone and the higher loss to follow-up in this group $(30 \%)$ versus the decompression and fusion group $(7 \%)$ is a limitation. Another weakness is the age differences between the groups. The age difference may account for the differences in outcomes. The outcomes selected were also non-validated, and may not be reliable. This study may also represent a best-case scenario of patients with degenerative scoliosis being treated with a decompression without fusion. This study was performed at a tertiary spine center with extensive experience in treating adult deformity. The biases in clinical judgment and decisions may be a factor in our findings.

\section{Clinical relevance and impact}

- Limiting a fusion to the decompressed regions only in patients with mild degenerative scoliosis, and stenosis in patients older than 65 years appears to stabilize the decompressed segments enough to prevent early recurrence of stenosis symptoms and curve progression.

- Decompression alone led to a higher rate of recurrence of stenotic symptoms.

- Age may play a role in the differences noted in our study groups.

\section{SUMMARY AND CONCLUSIONS}

Decompression and limited fusion of the decompressed segments in the setting of mild degenerative scoliosis $\left(<30^{\circ}\right)$ appears to be a more effective treatment for patients 65 years or older with symptoms of stenosis. 


\section{REFERENCES}

1. Marchesi DG, Aebi M (1992) Pedicle fixation devices in the treatment of adult lumbar scoliosis. Spine; 17 Suppl 8:304-309.

2. Simmons ED Jr, Simmons EH (1992) Spinal stenosis with scoliosis. Spine; 17 Suppl 6:117120.

3. Carreon LY, Puno RM, Dimar JR 2nd, et al (2003) Perioperative complications of posterior lumbar decompression and arthrodesis in older adults. $J$ Bone Joint Surg Am; 85A(11):2089-2092.

4. Ghiselli G, Wang JC, Bhatia NN, et al (2004) Adjacent segment degeneration in the lumbar spine. J Bone Joint Surg Am; 86-A(7):14971503.

5. Berven SH, Deviren V, Mitchell B, et al (2007) Operative management of degenerative scoliosis: an evidence-based approach to surgical strategies based on clinical and radiographic outcomes. Neurosurg Clin N Am; 18(2):261272.

6. Kostuik J, Bridwell KH, DeWald RL (1997) Adult scoliosis: the lumbar spine. The Textbook of Spinal Surgery. 2nd ed. Philadelphia: Lippincott-Raven, 767-775.

7. Grubb SA, Lipscomb HJ, Suh PB (1994) Results of surgical treatment of painful adult scoliosis. Spine; 19(14):1619-1627.

8. Park P, Garton HJ, Gala VC, et al (2004) Adjacent segment disease after lumbar or lumbosacral fusion: review of the literature. Spine; 29(17):1938-1944.

\section{EDITORIAL PERSPECTIVE}

The reviewers were unanimous in their assessment that the study by Daubs et al is very interesting with a highly relevant subject matter in light of an increasing aging population, substantial disability, and healthcare costs associated with the condition paraphrased as "degenerative lumbar scoliosis." As pointed out by the authors, there were concerns regarding inherent limitations of this study, especially revolving around the lack of homogeneity between the two-study populations. One group consisted largely of older patients in poor health treated with decompression alone; a second group consisted of relatively younger patients in good health treated with limited decompression and instrumented fusion. From a clinical perspective, it is unclear if this article is providing results that may help the reader decide how to treat a similar set of patients with one of the two alternatives. The study, however, unequivocally does show a higher complication and reoperation rate associated with the limited decompression group within their limited cohorts of 16 and 39 patients, and while patient factors should be kept in mind, this circumstance alone warrants a closer look at possible spine-related reasons for these reoperations regardless of patient covariables.

Other points to consider in the evaluation of this study are as follows:

- Aside from setting a curve limit with a Cobb angle of $30^{\circ}$, the authors did not address a number of other variables, such as presence or severity of lateral listhesis, presence of lateral spondylolisthesis, severity of facet pathology, history of prior surgery, or presence of sagittal malalignment. These are significant variables, which may have swayed the surgeons toward instrumented fusion. This would be a helpful addition to the understanding of curve severity and allow for a more differentiated review.

- Daubs et al relied on a non-standardized outcome measure similar to Odom's criteria, which is not as differentiated as ODI and VAS. Again, these are the confinements of a retrospective study and underscore the desirability to collect baseline outcome parameters on spine patients undergoing surgical treatment.

To their credit, the authors readily addressed and acknowledged these limitations. That said, the subject matter of degenerative scoliosis is of such pressing relevance, and starting with causation to symptom modulation is so poorly understood, that the conceptual merits of this study outweighs the methodological concerns. We ask that the readership consider these and engage in discussion through comments. Most important, the EBSJ Editorial staff wishes to thank Daubs and colleagues for taking this significant first step and raising important questions with this study. 